\title{
48. On a Regular Function, whose Real Part is Positive in a Unit Circle.
}

\author{
By Masatsugu TSUJI. \\ Mathematical Institute, Tokyo University. \\ (Coinm. by S. KakeYA, M.I.A., May, 1945.)
}

1. Carathéodory's theory ${ }^{1)}$ of positive harmonic functions in a unit circle atracted interests of many mathematicians ${ }^{D}$ and several proofs were given and the results were completed and now the main results stand in the following theorems. In this peper, I will give a simple proof, where the proof of Theorem I(I) is suggested by Szasz's paper!' and the proof of Theorem 1 (II) is the same as Schur's proof ${ }^{\mathrm{N}}$ essentially, but in a modified form.

Teeorem 1. Let $f(z)=\frac{a_{0}}{2}+\sum_{n=1}^{\infty} a_{n} z^{n}\left(a_{0}=\right.$ real $)$ be regular in $|z|<1$. Then (I)(Carathéodor'y ${ }^{1}$-Toeplitz). ${ }^{2)} \quad \Re f(z) \geqq 0$ in $|z|<1$, when and only when the Hermitian forms $H_{22}(x)=\sum_{0}^{n} a_{\mu-\nu} x_{\nu} \bar{x}_{\mu}\left(a_{-\nu}=\bar{a}_{\nu}\right)^{3)}$ are non-negative for $n=0,1,2, \ldots$ If all $H_{n}(x)$ are non-negative and $H_{0}(x), \ldots, H_{k-1}(x)$ are positive definite and $H_{k}(x)$ is pusitive semi-definite, then $f(z)$ is of the for $m$ :

$$
f(z)=\sum_{\nu=1}^{k} \frac{r_{\nu}}{2} \cdot \frac{1+\epsilon_{\nu} z}{1-\epsilon_{\nu} z},\left(r_{\nu}>0,\left|\epsilon_{\nu}\right|=1, \epsilon_{i} \neq \epsilon_{j}(i \neq j)\right),
$$

where $k$ is the rank of the infinite Hermitian matrix $H$ :

$$
H=\left(\begin{array}{l}
a_{0}, a_{1}, a_{1}, \ldots \\
\bar{a}_{1}, a_{0}, a_{1}, \ldots \\
\bar{a}_{3}, \bar{a}_{1}, a_{0}, \ldots \\
\ldots \ldots \ldots
\end{array}\right) .
$$

(II) (I. Schur).") If we put

1) C. Carathéodory: Über die Variabilitatsbereich der Fourierschen Konstanten von positiven harmonischen Funktionen. Rendiconti del circolo mat. Palermo. 32 (1911).

2) O. Toeplitz: Über die Fouriersche Entwicklung positiver Funktionen. Rendiconti del circolo mat. Palermo. 32 (1911). E. Fischer: Über das Carathéodorysche Problem. Rendiconti del circolo mat. Palermo. 32 (1911). I. Schur: Über potentreihen, die in Innern des Einheitskreises beschränkt sind. Crelle. 147 (1917). O. Szasz: Über harmonischen Funktionen und I Formen. Math. Zeits. 1 (1918). G. Szegö Über Funktionen mit positiver Realteil. Math. Ann. 99 (1928). F. Riesz: Über ein Problem des Herrn Carathéodory. Crelle 146 (1916).

3) In this paper, $\bar{a}$ means the conjugate complex of $a$. 


$$
\delta_{n}=\delta\left(a_{0}, a_{1}, \ldots, a_{n}\right)=\left|\begin{array}{ll}
a_{0}, a_{1}, & a_{1}, \ldots, a_{n} \\
\bar{a}_{1}, a_{0}, & a_{1}, \ldots, a_{n-1} \\
\ldots . . . . & \\
\bar{a}_{n}, \bar{a}_{n-1}, & \bar{a}_{n-2}, \ldots, a_{0}
\end{array}\right|,
$$

then $\Re f(z) \geqq 0$ in $|z|<\mathbf{1}$, when and only when (i) $\delta_{n}>\mathbf{0}$ for all.n or (ii) $\delta_{0}>0, \delta_{1}>0, \ldots, \delta_{k-1}>0, \delta_{k}=\delta_{k+1}=\ldots=0$ for some $k$. This case occur's only when $f(z)$ is $\& f$ the form (1).

Theorem 2. (Carathéodory).4) Let $a_{0}, a_{1}, \ldots, a_{n}\left(a_{0}=r e a l\right)$ be $n+1$ complex numbers, such that $H_{n}(x)=\int_{0}^{n} \alpha_{\mu-\nu} x_{\nu} \bar{x}_{\mu}$ is non-negative. Then there exists a regular function $f(z)$ in $|z|<1$, such that $\Re f(z) \geqq 0$ in $|z|<1$ and

$$
f(z)=\frac{a_{0}}{2}+a_{1} z+\ldots+a_{n} z^{n}\left(\bmod . z^{n+1}\right) .
$$

If $H_{n}(x)$ is positive semi-definite, then such $f(z)$ is unique and is of the form (1), where $k \leqq n$.

For the proof, we use the followong theorems.

Theorem A. (Fejer $) \cdot{ }^{D} \quad$ Let $\tau(\boldsymbol{\varphi})=\lambda_{0}+\sum_{\nu=1}^{2}\left(\lambda_{\nu} \cos \nu \boldsymbol{\varphi}+\mu_{\nu} \sin \nu \boldsymbol{\varphi}\right) \geqq 0$ in $[0,2 \pi]$. Then $\tau(\varphi)$ can be expressed in the form:

$$
\tau(\boldsymbol{\varphi})=\left|\gamma_{0}+\gamma_{1} e^{i \varphi}+\ldots+\gamma_{n} e^{i n \varphi}\right|^{2} \text {. }
$$

Theorem B. (I. Schur). ${ }^{6} \quad$ Let $A=\sum_{1}^{n} a_{\nu_{\mu}} x_{\nu} \bar{x}_{\mu}$ be a Hermitian form, such that

$$
a^{\prime} \leqq A \leqq a \text { for }\left|x_{1}\right|^{2}+\left|x_{2}\right|^{2}+\ldots+\left|x_{n}\right|^{2}=1
$$

and $B=\sum_{1}^{n} b_{\nu_{\mu} x_{\nu}{ }_{2} \bar{x}_{\mu}}$ be a non-negative Hermitian form,

$$
b^{\prime}=\operatorname{Min} .\left(b_{11}, b_{22}, \ldots, b_{n 2}\right), b=\operatorname{Max} .\left(b_{11}, b_{22}, \ldots b_{n n}\right) .
$$

Then

$$
\begin{gathered}
a^{\prime} b^{\prime} \leqq \sum_{1}^{n} a_{\nu_{\mu}} b_{\nu_{\mu}} x_{\nu} \bar{x}_{\mu} \leqq a b \\
\text { for } \quad\left|x_{1}\right|^{2}+\left|x_{1}\right|^{2}+\ldots+\left|x_{n}\right|^{2}=\overline{1} .
\end{gathered}
$$

4) C. Carathéodory. 1.c. (1). I. Schur: Über einen Satz von C. Carathéodory. Berliner Ber. 1912. G. Frobenius: Ableitung eines Satzes von Carathéodory aus einer Formel von Kronecker. Berliner Ber. 1912.

5) L. Féjér : Über trigonometrische Polynome. Crelle 146 (1916).

6) I. Schur: Bemerkungen zur Theorie der beschränkten Bilinearformen mit unendlich vielen Veranderlichen. Crelle 140 (1911). O. Szasz. 1.c. (2). 
No. 5.] On a regular Function, whosè real Part is positive in a Unit Círcle.

2. Proof of Theorem 1(I).

(i ) Let $\Re f(z) \geqq 0$ in $|z|<1$, then by Herglotz's theorem, $f(z)$ can be expressed by

$$
f(z)=\frac{a_{0}}{2}+\sum_{n=1}^{\infty} a_{n} z^{n}=\frac{1}{2 \pi} \int_{0}^{2 \pi} \frac{e^{i \varphi}+z}{e^{i \varphi}-z} d x(\varphi),
$$

where $\chi(\varphi)$ is a non-decreasing function of $\boldsymbol{\phi}$, so that

$$
a_{n 2}=\frac{1}{\pi} \int_{0}^{2 \pi} e^{-i n \varphi} d \chi(\varphi)(n=0,1,2, \ldots)
$$

Hence

$$
\begin{array}{r}
H_{n}(x)=\sum_{0}^{n} a_{\mu-\nu} x_{\nu} \bar{x}_{\mu}=\frac{1}{\pi} \int_{0}^{2 \pi}\left|x_{0}+x_{1} e^{i \varphi}+\ldots+x_{n} e^{i n \varphi}\right|^{2} d \chi \geqq 0 . \\
(n=0,1,2, \ldots)
\end{array}
$$

(ii) Next we will prove that $\Re f(z) \geqq 0$ in $|z|<1$, if all $H_{n}(x)$ are nonnegative. Since for $|z|<\rho<1$,

$$
f(z)=\frac{a_{0}}{2}+\sum_{n=1}^{\infty} a_{n} z^{n}=\frac{1}{2 \pi} \int_{0}^{2 \pi} \Re\left(\rho e^{i \varphi}\right) \frac{\rho e^{i \varphi}+z}{\rho e^{i \varphi}-z} d \boldsymbol{\phi}
$$

we have

$$
a_{n} \rho^{n}=\frac{1}{\pi} \int_{0}^{2 \pi} f\left(\rho e^{i \varphi}\right) e^{-i n \varphi} d \boldsymbol{\phi}(n=0,1,2, \ldots)
$$

so that

$$
\begin{aligned}
& H_{2}^{(\mathrm{P})}(x)=\sum_{0}^{n} a_{\mu-\nu} \rho^{|\mu-\nu|} \alpha_{\nu} \bar{x}_{\mu}= \\
& \quad \frac{1}{\pi} \int_{0}^{2 \pi} \Re f\left(\rho e^{i \varphi}\right)\left|x_{0}+x_{1} e^{i \varphi}+\ldots+x_{\eta} e^{i n \varphi}\right|^{2} d \varphi .
\end{aligned}
$$

Let

$$
g(z)=\frac{1}{2}+\sum_{n=1}^{\infty} z^{n}=\frac{1+z}{2(1-z)}, \text { then } \Re g(z)=\frac{1-|z|^{2}}{2|1-z|^{2}}>0 \text { in }|z|<1 .
$$

Hence by (8),

$$
B=\sum_{0}^{n} \rho^{|\mu-\nu|} x_{\nu} \bar{\partial}_{\mu}=\frac{1}{\pi} \int_{0}^{2 \pi} \Re g\left(\rho e^{i \varphi}\right)\left|x_{0}+x_{1} e^{i \varphi}+\ldots+x_{n} e^{i n \varphi}\right| 2 d \boldsymbol{\varphi} \geqq 0 .
$$

We put for $\left|x_{0}\right|^{2}+\left.\right|^{2}+\ldots+\left|x_{n 2}\right|^{2}=1$,

$$
\begin{gathered}
g_{n}=\operatorname{Min} . H_{n}(x), G_{n}=\operatorname{Max} \cdot H_{n}(x), \\
g_{n}^{(P)}=\operatorname{Min} . H_{n}^{(P)}(x), G_{n}^{(p)}=\operatorname{Max} . H_{n}^{(P)}(x) .
\end{gathered}
$$

Since $g_{0}^{(\rho)} \geqq g_{1}^{(\rho)} \geqq \ldots \geqq g_{12}^{(\rho)}, \ldots G_{0}^{(p)} \leqq G_{1}^{(\rho)} \leqq \ldots \leqq G_{n}^{(p)}$, let

$$
\lim _{n \rightarrow \infty} g_{n}^{(p)}=g^{(p)}, \lim _{n \rightarrow \infty} G_{n}^{(p)}=G^{(p)} \text {. }
$$


We apply Theorem $B$ on $A=H_{n}(x)=\sum_{0}^{n} a_{\mu-\nu} x_{\nu} \bar{x}_{\mu}, B=\sum_{0}^{n} \rho^{\prime \mu-\nu_{i}} x_{\nu} \bar{x}_{\mu}$, then since $H_{n}(x) \geqq 0$ and $b_{11}=b_{: 2}=\ldots=b_{n n}=1$, we have $0 \leqq g_{n} \leqq g_{n 2}^{(\mathrm{P})} \leqq G_{n}^{(\mathrm{P})} \leqq G_{n}$, so that

$$
0 \leqq g^{(P)} \leqq g_{2 i}^{(P)} \leqq G_{22}^{(p)} \leqq G^{(p)}
$$

Let $\rho e^{i \rho_{0}}$ be, any point on $|z|=\rho$, then $\left|\Re f\left(\rho e^{i \varphi}\right)-\Re f\left(\rho e^{i \varphi_{0}}\right)\right|<\epsilon$ for $\left|\boldsymbol{\varphi}-\boldsymbol{\varphi}_{0}\right| \leqq \delta$. We define a positive continuous function $g(\boldsymbol{\varphi})$ in $[0,2 \pi]$ by the following conditions: (i) $\int_{0}^{2 \pi} g(\varphi) d \varphi=2 \pi$, (ii) $g(\varphi)=$ const. $=M(>0$ ) in $\left|\boldsymbol{\varphi}-\boldsymbol{\varphi}_{0}\right| \leqq \delta$ and is a linear function in $\left[\boldsymbol{\varphi}_{0}-\delta-\delta^{\prime}, \phi_{0}-\delta\right]$ and $\left[\boldsymbol{\varphi}_{0}+\delta, \boldsymbol{\varphi}_{0}+\delta+\delta^{\prime}\right]$, such that $g\left(\boldsymbol{\varphi}_{0}-\delta-\delta^{\prime}\right)=\eta, g\left(\boldsymbol{\varphi}_{0}-\delta\right)=M, g\left(\boldsymbol{\varphi}_{0}+\delta\right)$ $=M, g\left(\boldsymbol{\varphi}_{0}+\delta+\delta^{\prime}\right)=\eta(\eta>0)$ and $g(\boldsymbol{\varphi})=$ const. $^{\prime}=\eta$ in the remaining part of $[0,2 \pi]$, where we take $M$ so large and $\delta^{\prime}, \eta$ so small, that

$$
\int_{J} g(\boldsymbol{\varphi}) d \boldsymbol{\rho}<\epsilon, \text { so that } \int_{\varphi 0-\delta}^{\varphi 0+\delta} g(\boldsymbol{\varphi}) d \boldsymbol{\varphi}=2 \pi-0(\epsilon),
$$

where $J$ is the complementary set of $\left|\boldsymbol{\varphi}-\boldsymbol{\varphi}_{0}\right| \leqq \delta$ in $[0,2 \pi]$.

Now we approximate $g(\boldsymbol{\varphi})$ by a trigonometrical polynomial $\tau(\boldsymbol{\varphi})$ of order $n$, such that $|g(\varphi)-\tau(\varphi)|<\epsilon_{1}(<\eta)$ in $[0,2 \pi]$, then $\tau(\phi)>0$ in $[0,2 \pi]$. Hence by Theorem $A, \tau(\varphi)=\left|x_{0}+x_{1} e^{s_{\varphi}}+\ldots+x_{n} e^{i_{n} \varphi}\right|^{2}$ with suitable $x_{0}, x_{1}, \ldots, x_{20}$ Then

$2 \pi\left(\left|x_{0}\right|^{2}+\left|x_{1}\right|^{2}+\ldots+\left|x_{z_{2}}\right|^{2}\right)=\int_{0}^{2 \pi} \tau(\varphi) d \varphi=\int_{0}^{2 \pi} g(\varphi) d \varphi+0\left(\epsilon_{1}\right)=2 \pi+0\left(\epsilon_{1}\right)$, so that $\left|x_{0}\right|^{2}+\left|x_{1}\right|^{2}+\ldots+\left|x_{n}\right|^{2}=1+0\left(\epsilon_{1}\right)$.

From (8), (12), (13),

$$
\begin{aligned}
& 0 \leqq g^{(\rho)} \cdot\left(\left|x_{0}\right|^{2}+\left|x_{1}\right|^{2}+\ldots+\left|x_{i n}\right|^{2}\right) \leqq H_{i 0}^{(\rho)}(x)=\frac{1}{\pi} \int_{0}^{2 \pi} \Re f\left(\rho e^{i \varphi}\right) \tau(\phi) d \phi \\
& =\frac{1}{\pi} \int_{0}^{i \pi} \Re f\left(\rho e^{i \varphi}\right) g(\varphi) d \varphi+0\left(\epsilon_{1}\right)=\frac{1}{\pi} \int_{\varphi 0-\delta}^{\varphi 0+\delta} \Re f\left(\rho e^{i \varphi}\right) g(\varphi) d \varphi \\
& +\frac{1}{\pi} \int_{J} \Re f\left(\rho e^{i \varphi}\right) g(\varphi) d \varphi+0\left(\epsilon_{1}\right)=\frac{\Re f\left(\rho e^{i \varphi_{0}}\right)}{\pi} \int_{\varphi_{0}-\delta}^{\varphi \varphi^{+\delta}} g(\bar{\varphi}) d \varphi+0(\epsilon) \\
& +0(1) \int g(\varphi) d \varphi+0\left(\epsilon_{1}\right)=2 \Re f\left(\rho e^{i \varphi_{0}}\right)+0(\epsilon)+0\left(\epsilon_{1}\right)
\end{aligned}
$$

Making $\epsilon \rightarrow 0, \epsilon_{1} \rightarrow 0$ we have

$$
0 \leqq g^{(\rho)} \leqq 2 \Re f\left(\rho e^{i \rho 0}\right)
$$

Hence $\Re f(z) \geqq 0$ in $|z|<1$, q.e.d.

(iii) Suppose that all $H_{22}(x)$ are non-negative and $H_{0}(x), H_{1}(x), \ldots$, $H_{k-1}(x)$ are positive definite and $H_{k}(x)$ is positive semi-definite. Then there exists $x_{0}^{\prime}, x_{1}^{\prime}, \ldots, x_{k}^{\prime}\left(\left|x_{0}^{\prime}\right|^{2}+\left|x_{1}^{\prime}\right|^{2}+\ldots+\left|x_{k}^{\prime}\right|^{2}=1\right)$, such that $H_{k}\left(x^{\prime}\right)=0$ Since by (ii) $\mathscr{A} f(z) \geqq 0$ in $|z|<1$, we have by (5), 
No. 5.] On a regular Function, whose real Part is positive in a Unit Circle.

$$
\int_{0}^{2 \pi}\left|x_{0}^{\prime}+x_{1}^{\prime} e^{i \varphi}+\ldots+x_{k}^{\prime} e^{i k k_{\rho}}\right|^{2} d \chi=0
$$

If $\chi(\varphi)$ is increasing at $\phi=\varphi_{0}$ and $x_{0}^{\prime}+x_{1}^{\prime} e^{i \varphi_{0}}+\ldots+x_{k}^{\prime} e^{\alpha k p_{0}} \neq 0$, then $\left|x_{0}^{\prime}+x_{1}^{\prime} e^{i \varphi}+\ldots+x_{k}^{\prime} e^{i k \varphi}\right| \geqq \eta>0$ for $\left|\varphi-\varphi_{0}\right| \leqq \delta$, so that $\int_{0}^{2 \pi} \mid x_{0}^{\prime}+x_{1}^{\prime} e^{i \varphi}+\ldots+$ $\left.x_{k} e^{i k \varphi}\right|^{2} d \varphi>0$, which contradicts to (15). Hence if $\chi(\varphi)$ is increasing at $\varphi=\varphi_{0}$, then $x_{11}^{\prime}+x_{1}^{\prime} e^{i \varphi_{0}}+\ldots+x_{k}^{\prime} e^{i k \varphi_{0}}=0$. Since $x_{0}^{\prime}+x^{\prime} z+\ldots+x_{k}^{\prime} z^{k}=0$ has at most $k$ roots on $|z|=1, \chi(\varphi)$ is increasing at $\varphi_{1}, \ldots, \varphi_{j}(j \leqq k)$ and is constant outside $\boldsymbol{\phi}_{v}$, so that by (5),

$$
\begin{array}{r}
H_{n}(x)=\sum_{\nu=1}^{j} r_{\nu}\left|x_{0}+x_{1} e^{i \varphi_{\nu}}+\ldots+x_{n} e^{i n \rho_{\nu}}\right|^{2} \quad\left(r_{\nu}=\frac{d \chi\left(\varphi_{\nu}\right)}{\pi}>0\right), \\
(n=0,1,2, \ldots) .
\end{array}
$$

If $j \leqq k-1$, then a system of linear equations:

$$
x_{0}+x_{1} e^{i \varphi_{\nu}}+\ldots+x_{k-1} e^{i(k-1) \varphi_{\nu}}=0 \quad(\nu=1,2, \ldots, j)
$$

has a solution $x_{0}^{\prime \prime}, x_{1}^{\prime \prime}, \ldots, x_{k-1}^{\prime \prime}$, such that $\left|x_{0}^{\prime \prime}\right|^{2}+\left|x_{1}^{\prime \prime}\right|^{2}+\ldots+\left|x_{k-1}^{\prime \prime}\right|^{2}=1$. Then $H_{k-1}\left(x^{\prime \prime}\right)=0$, which contradicts the hypothesis. Hence $j=k$, so that by (3),

$$
f(z)=\sum_{\nu=1}^{k} \frac{r_{\nu}}{2} \cdot \frac{1+\epsilon_{\nu} z}{1-\epsilon_{\nu} z}\left(\epsilon_{\nu}=e^{-i \varphi_{\nu}}\right)
$$

hence

$$
\begin{gathered}
a_{n}=r_{1} \epsilon_{1}^{n}+\ldots+r_{k} \epsilon_{k}^{n}, \\
H_{n}(x)=\sum_{\nu=1}^{k} v_{\nu}\left|x_{0}+x_{1} \epsilon_{\nu}^{-1}+\ldots+x_{\gamma_{2}} \epsilon_{\nu}^{-n}\right|^{2} . \\
\quad(n=0,1,2, \ldots)
\end{gathered}
$$

From (17), we see easily that $k$ is the rank of $H$.

Conversely, if $f(z)$ is of the form $(1)$, then $H_{0}(x), \ldots, H_{k-1}(x)$ are positive definite and $H_{k}(x)$ is positive semi-definite.

3. A Lemma to the proof of Theorem 1 (II).

Let $f(z)=\frac{a_{0}}{2}+\sum_{n=1}^{\infty} a_{22} z^{n}$ be regular for $: z \mid<1$ and suppose that $a_{0}>0$, we define $f_{1}(z)$ by the following relations:

$$
\begin{aligned}
& \varphi(z)=\frac{1-f(z)}{1+f(z)}=\alpha_{0}+\alpha_{1} z+\alpha_{2} z^{2}+\ldots, \alpha_{0}=\frac{2-a_{0}}{2+a_{0}},\left(\left|\alpha_{0}\right|<1\right), \\
& \varphi_{1}(z)=\frac{\epsilon}{z} \cdot \frac{\varphi(z)-\alpha_{0}}{1-\alpha_{0} \varphi(z)},(|\epsilon|=1), \\
& f_{1}(z)=\frac{1-\varphi_{1}(z)}{\Gamma+\varphi_{1}(z)}=\frac{\left(a_{0}+\epsilon a_{1}\right)+\left(a_{1}+\epsilon a_{2}\right) z+\ldots+\left(a_{n}+\epsilon a_{n+1}\right) z^{n}+\ldots}{\left(a_{0}-\epsilon a_{1}\right)+\left(a_{1}-\epsilon a_{2}\right) z+\ldots+\left(a_{n}-\epsilon a_{n+1}\right) z^{n}+\ldots}
\end{aligned}
$$




$$
=\frac{c_{0}}{2}+c_{1} z+c_{2} z^{2}+\ldots, c_{0}=2 \frac{a_{0}+\epsilon a_{1}}{a_{0}-\epsilon a_{1}},
$$

where we determine $\epsilon(|\epsilon|=1)$, such that $a_{0}-\epsilon a_{1} \neq 0$ and $c_{0}$ is real. That this is always possible is seen as follows. If $a_{1}=0$, then we take $\epsilon=1$ and if $a_{1} \neq 0$, we take as $\epsilon$ one of solutions of $\epsilon^{2}=\frac{\bar{a}_{1}}{a_{1}}$, such that $a_{0}-\epsilon a_{1} \neq 0$, which is possible, since if $a_{0}-\epsilon a_{1}=0, a_{0}+\epsilon a_{1}=0$, then we would have $a_{0}=0, a_{1}=0$, which contradicts to $a_{0}>0 . \quad c_{3}$ is real since $\epsilon^{2}=\frac{\bar{a}_{1}}{a_{1}}$. Then we have

$$
\text { Lemma. } \delta\left(c_{0}, c_{1}, \ldots, c_{\nu}\right)=\frac{2^{\nu+1} a_{0}^{\nu}}{\left|a_{0}-\epsilon a_{1}\right|^{2 \nu+2}} \delta\left(a_{0}, a_{1}, \ldots, a_{\nu+1}\right) .
$$

Proof. Let for any matrix $A$, we denote its $\nu$-th section by $A_{\nu}$, which is a matrix formed with elements of $A$ lying in the first $\nu$ rows and first $\nu$ columns and put $\left|A_{\nu}\right|=\operatorname{det} . A_{\nu}$. Let

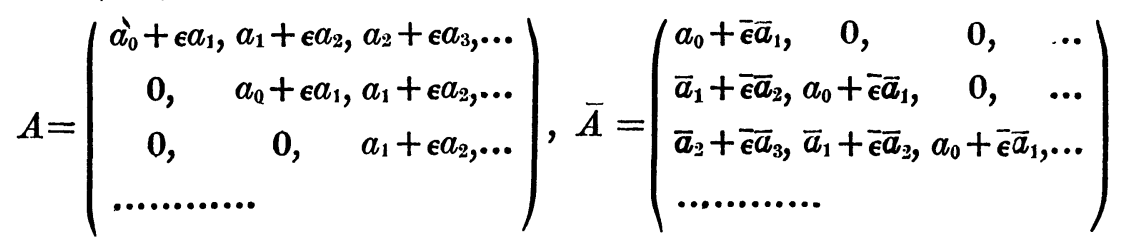

be infinite matrices and $B, \bar{B}^{\prime}, C, \bar{C}^{\prime}$ be infinite matrices similarly formed with $a_{0}-\epsilon a_{1}, a_{1}-\epsilon a_{2}, \ldots$ and $\frac{c_{0}}{2}, c_{1}, c_{2}, \ldots$ respectively.

Let

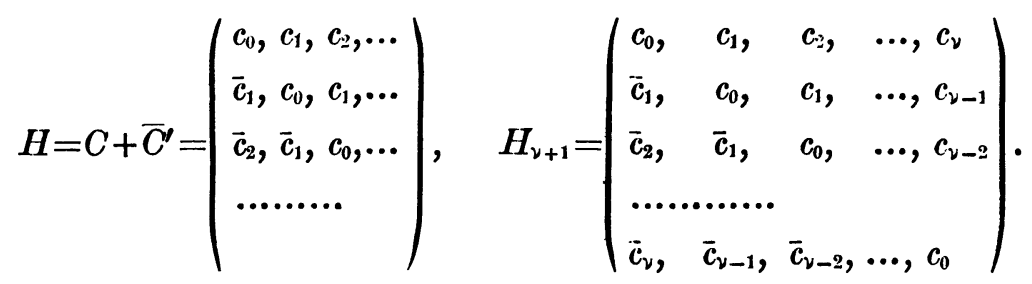

Then $\left|H_{\nu+1}\right|=\delta\left(c_{0}, c_{1}, \ldots c_{\nu}\right)$. From (18), we have $\frac{A}{B}=C, A=C B, A B^{-1}=C^{i}$, so that $H=A B^{-1}+\left(\overline{B^{\prime}}\right)^{-1} \overline{A^{\prime}}$, hence $\overline{B^{\prime}} H B=\overline{B^{\prime}} A+\overline{A^{\prime}} B$.

Now

$$
\bar{B}^{\prime} A+\bar{A}^{\prime} B=\left(\begin{array}{l}
2\left(a_{0} a_{0}-\bar{a}_{1} a_{T}\right), 2\left(a_{0} a_{1}-\bar{a}_{1} a_{2}\right), 2\left(a_{0} a_{2}-\bar{a}_{1} a_{3}\right), \ldots \\
2\left(a_{0} \bar{a}_{1}-\bar{a}_{2} a_{1}\right), 2\left(a_{0} a_{0}-\bar{a}_{2} a_{2}\right), 2\left(a_{0} a_{1}-\bar{a}_{2} a_{3}\right), \ldots \\
2\left(a_{0} \bar{a}_{2}-\bar{a}_{3} a_{1}\right), 2\left(a_{0} \bar{a}_{1}-\bar{a}_{3} a_{2}\right), 2\left(a_{0} a_{0}-\bar{a}_{3} a_{3}\right), \ldots \\
\ldots \ldots \ldots \ldots \ldots \ldots
\end{array}\right) .
$$

7) I. Schur. l. c. (2) 
Then

$$
\begin{aligned}
& \left|\left(\bar{B}^{\prime} A+\bar{A}^{\prime} B\right)_{\nu+1}\right|=\left|\left(\bar{B}^{\prime} H B\right)_{\nu+1}\right|=\left|\bar{B}_{\nu+1}^{\prime}\right|\left|H_{\nu+1}\right|\left|B_{\nu+1}\right| \\
& \quad=\left|a_{0}-\epsilon a_{1}\right|^{2 v+2}\left|H_{\nu+1}\right|=\left|a_{0}-\epsilon a_{1}\right|^{2 \nu+2} \delta\left(c_{0}, c_{1}, \ldots c_{\nu}\right) .
\end{aligned}
$$

We apply Sylvester's theorem on the upper left corner element $a_{0}$ of $\delta\left(a_{0}, a_{1}, \ldots, a_{\nu+1}\right)$, then we have $\left|\left(B^{\prime} A+A^{\prime} B\right)_{\nu+1}\right|=2^{\nu+1} a_{0}^{\nu} \delta\left(a_{0}, a, \ldots a_{\nu+1}\right)$, so that

$$
\delta\left(c_{0}, c_{1}, \ldots, c_{\nu}\right)=\frac{2^{\nu+1} a_{0}^{\nu}}{\left|a_{0}-\epsilon a_{1}\right|^{2 \nu+2}} \delta\left(a_{0}, a_{1}, \ldots, a_{\nu+1}\right) .
$$

\section{Proof of Theorem 1 (II).}

If $\Re f(z) \geqq 0$ in $|z|<1$, then by Theorem $1(\mathrm{I})$, all $H_{n}(x)=\sum_{0}^{n} a_{\mu-\nu} x_{\nu} \bar{x}_{\mu}$ are non-negative, so that (1) $\delta_{n}>0$ for all $n$ or (ii) $\delta_{0}>0, \delta_{1}>0, \ldots, \delta_{k-1}>0$, $\delta_{k}=\delta_{k+1}=\ldots=0$ for some $k$. Conversely, if $\delta_{n}>0$ for all $n$, then $H_{n}(x)$ are positive definite, so that by Theorem $1(\mathrm{I}), \Re f(z) \geqq 0$ in $|z|<1$. Next we will prove that $\Re f(z) \geqq 0$ in $|z|<1$ in case (ii).

First we remark that if all $\delta_{n 2}=0$, then all $a_{n 2}=0$. For, from $\delta_{0}=0$, we have $a_{0}=0$. Suppose that $a_{0}=a_{1}=\ldots=a_{k-1}=0$ be proved, then $\delta\left(a_{0}, a_{1}, \ldots, a_{2 k-1}\right)$ $=\delta\left(0,0, \ldots 0, a_{k j} \ldots, a_{2 k-1}\right)=(-1)^{k}\left|a_{k}\right|^{2 k}=0, a_{k}=0$. Hence by induction, all $a_{n}=0$, so that $f(z) \equiv 0, \Re f(z)=0$ in $|z|<1$.

Suppose by induction that it is proved that $\Re f(z) \geqq 0$ in $|z|<1$, if $\delta_{0}>0$, $\delta_{1}>0, \ldots, \delta_{k-2}>0, \delta_{k-1}=\ldots=0$, the case $k=1$ being proved above, and let $\delta\left(a_{0}\right)>0, \delta\left(a_{0}, a_{1}\right)>0, \ldots, \delta\left(a_{0}, a_{1}, \ldots, a_{k-1}\right)>0, \delta\left(a_{0}, a_{1}, \ldots, a_{k}\right)=\ldots=0$.

$f_{1}(z)=\frac{c_{0}}{2}+c_{1} z+c_{2} z^{2}+\ldots$ be the function defined in the lemma, then by the lemma, we have $\delta\left(c_{0}\right)>0, \delta\left(c_{0}, c_{T}\right)>0, \ldots, \delta\left(c_{0}, c_{1}, \ldots, c_{k-2}\right)>0, \delta\left(c_{0}, c_{1}, \ldots, c_{k-1}\right)$ $=\ldots=0$, so that by induction, $\Re f_{1}(z) \geqq 0$ in $|z|<1$. From this we conclude casily that $\Re f(z) \geqq 0$ in $|z|<1$. Since $\Re f(z) \geqq 0$ in $|z|<1$, vall $H_{n}(x)$ $=\sum_{0}^{n} a_{\mu-\nu} x_{\nu} \bar{x}_{\mu}$ are non-negative and since $\delta\left(a_{0}\right)>, \ldots, \delta\left(a_{0}, a, \ldots a_{k-1}\right)>0$, $\delta\left(a_{0}, a_{1}, \ldots a_{k}\right)=\ldots=0, H_{0}(x), \ldots, H_{k-1}(x)$ are positive definite and $H_{k}(x)$ is positive semi-definite, so that $f(z)$ is of the form (1).

\section{Proof of Theorem 2.}

We consider $\left(a_{0}, a_{1}, \ldots, a_{n 2}\right)$ as a point in a $2 n+2$-dimensional space and consider with Caratheodory a domain $\Re_{n}$ :

$$
\mathfrak{\Omega}_{22}: \delta\left(a_{11}\right)>0, \delta\left(a_{0}, a_{1}\right)>0, \ldots, \delta\left(a_{0}, a_{1}, \ldots, a_{22}\right)>0 .
$$

Then $H_{n}(x)=\sum_{0}^{n} a_{\mu-\nu} x_{\nu} \bar{x}_{\mu}$ is positive definite. From this we see easily that $\Omega_{n z}$ is a convex domain. Its boundary consists of points:

$$
\delta\left(a_{0}\right)>0, \delta\left(a_{0}, a_{1}\right)>0, \ldots, \delta\left(a_{0}, a_{1}, \ldots, x_{k-1}\right)>0, \delta\left(a_{0}, a_{1}, \ldots, a_{k}\right)=\ldots=0
$$


for some $k \leqq n$, since the boundary point corresponds to a positive semi-definite form $H_{n}(x)$. Suppose that $H_{n}(x)$ is non-negative. Then $\left(a_{0}, a_{1}, \ldots, a_{n}\right)$ lies in or on the boundary of $\Omega_{n}$, so that there exists in its $\epsilon$-neighbourhood a point $\left(a_{n}^{\prime}, a_{1}^{\prime}, \ldots, a_{n}^{\prime}\right)$ which lies in $\Re_{n}$, so that $\delta\left(a_{0}^{\prime}\right)>0, \delta_{1}^{\prime}\left(a_{0}^{\prime}, a_{1}^{\prime}\right)>0, \ldots$ $\delta_{n}^{\prime}=\delta\left(a_{0}^{\prime}, a_{1}^{\prime}, \ldots, a_{n}^{\prime}\right)>0$. Let $F(z)=\delta\left(a_{0}^{\prime}, a_{1}^{\prime}, \ldots a_{n}^{\prime}, z\right)=A \bar{z}+B z+B \bar{z}+C$, where $A, C$ are real.

Then $A=-\delta_{n-1}^{\prime} \neq 0$. Since by Jacobi's theorem, $\left|\begin{array}{l}\delta_{n z}^{\prime}, B \\ \bar{B}, \delta_{n}^{\prime}\end{array}\right|=\delta_{n-1}^{\prime} C=-A C$, $|B|^{2}-A C=\delta_{n 2}^{\prime 2}>0, F(z)=0$ is a real circle with a radius $r^{*}=\sqrt{|B|^{2}}-\bar{A} \bar{C}$. Hence there exists $a_{n+1}^{\prime}$, such that $F\left(a_{n+1}^{\prime}\right)=0$. Then $\left(a_{0}^{\prime}, a_{1}^{\prime}, \ldots, a_{n+1}^{\prime}\right)$ belongs to the boundary of $\Re_{2+1}$, so that $\left|\begin{array}{l}a_{0}^{\prime}, a_{n+1}^{\prime} \\ \bar{a}_{n+1}^{\prime}, a_{0}^{\prime}\end{array}\right| \geqq 0,\left|a_{n+1}^{\prime}\right| \leqq\left|a_{0}^{\prime}\right|$. Hence if we make $a_{0}^{\prime} \rightarrow a_{0}, \ldots, a_{n}^{\prime} \rightarrow a_{n}$, then the crresponding $a_{n+1}^{\prime}$ are bounded, so that we can select a convergent sequence from $a_{n+1}^{\prime}$, such that $a_{n+1}^{\prime} \rightarrow a_{n+1}$, then $\left(a_{0}, a_{1}, \ldots, a_{n+1}\right)$ belongs to the boundary of $\Re_{n+1}$, so that the corresponding Hermi tian form $H_{n+1}(x)$ is positive semi-definite. Similarly we can find $a_{n+2}, a_{n+3}$, $\ldots$, such that $H_{n+2}(x), H_{n+\varepsilon}(x), \ldots$ are pisitive semi-definite, so that $\left|a_{\psi}\right| \leqq a_{0}$ $(\nu=1,2, \ldots)$. Hence $f(z)=\frac{a_{n}}{2}+\sum_{n=1}^{\infty} a_{n} z^{n}=\frac{a_{0}}{2}+a_{1} z+\ldots+a_{n} z^{n}\left(\bmod . z^{n+1}\right)$ is regular in $: z \mid<1$ and by Theorem $1(I), \Re f(z) \geqq 0$ in $|z|<1$.

Next we will prove that if $H_{n}(x)$ is postive semi-definite, such $f(x)$ is unique. Suppose that $H_{0}(x), H_{1}(x), \ldots, H_{k-1}(x)$ are positive definite and $H_{k}(x)$ $(k \leqq n)$ is positive semi-definite, then by Theorem $1(I), f(z)$ is of the form (1), so that $a_{\nu}=r_{1} \epsilon_{1}^{\sigma}+\ldots+r_{k} \epsilon_{k}^{\nu}(\nu=0,1,2, \ldots)$. Hence $\epsilon_{1}, \ldots, \epsilon_{k}$ are roots of the equation:

$$
\begin{aligned}
& F_{k}(x)=\left|\begin{array}{ccc}
a_{0}, & a_{1}, & \ldots, a_{k-1}, a^{k} \\
\bar{a}_{1}, & a_{0}, & \ldots, a_{k-2}, a_{k-1} \\
\ldots \ldots \ldots \ldots & \\
\bar{a}_{k-1}, & \bar{a}_{k-2}, & \ldots, a_{0}, \quad a_{1} \\
1, & x, \quad \ldots, x^{k-1}, x^{k}
\end{array}\right| \\
& =\left|\begin{array}{llll}
r_{1}, & r_{2}, & \ldots, r_{k}, & 0 \\
r_{1} \varepsilon_{1}^{-1}, & r_{2} \varepsilon_{2}^{-1} & \ldots, r_{k} \varepsilon_{k}^{-1}, & 0 \\
\ldots \ldots . . . . & \\
r_{1} \varepsilon_{1}^{-k+1}, & r_{2} \varepsilon_{2}^{-k+1}, \ldots, r_{k}^{*} \varepsilon_{k}^{-k+1}, & 0 \\
0, & 0, & \ldots, 0, & 1
\end{array}\right| \cdot\left|\begin{array}{l}
1, \varepsilon_{1}, \ldots, \varepsilon_{1}^{k} \\
\ldots \ldots . \\
1,-\varepsilon_{k}, \ldots, \varepsilon_{k}^{k-1} \\
1, x, \ldots, x^{k}
\end{array}\right|=\delta\left(a_{0}, a_{k}, \ldots a_{k-1}\right) x^{k}+\ldots=0,
\end{aligned}
$$

so that $\varepsilon_{1}, \ldots, \varepsilon_{k}$ are unique and $r_{1} \ldots, r_{k}$ are unique, being the solution of a system of linear equations: $\quad a_{\nu}=r_{1} \varepsilon_{1}^{\nu}+\ldots+r_{k} s_{k}^{\nu}(\nu=0,1,2, \ldots, k-1)$. Hence $f(z)$ is unique. 
No. 5.] On a regular Function, whose real Part is positive in a Unit Circle.

5. The original proof of Theorem 2 depends on the following

Theorem 3 (Carathéodory). ${ }^{8)} \quad$ Let $a_{1}, a_{3}, \ldots, a_{n}$ be any given n complex numbers, then $a_{2}(\nu=1,2, \ldots, n)$ can be expressed in the for $m$ :

$$
a_{\nu}=r_{1} \varepsilon_{1}^{\nu}+\ldots+r_{k} \varepsilon_{k}^{\nu}\left(k \leqq n, r_{j}>0,\left|\varepsilon_{j}\right|=1, j=1,2, \ldots, k\right)
$$

and such $k, r_{j}, \varepsilon_{j}$ are unique.

This can be proved simply as follows. Since all the roots of the equation $\delta\left(x, a_{1}, \ldots, a_{n}\right)=0$ are real, let $a_{0}$ be its greatest root, then all characteristic numbers of the Hermitian form $H_{n}(x)=\sum_{0}^{n} a_{\mu-\nu} x_{\nu} \bar{x}_{\mu}$ are non-negative, hence $H_{n}(x)$ is positive semi-definite, since $\delta\left(a_{0}, a_{1}, \ldots x_{n}\right)=0$. Hence by Theorem 2, there exists a unique

$f(z)=\frac{a_{0}}{2}+a_{1} z+\ldots+a_{n} z^{n}\left(\bmod . z^{n+1}\right)=\sum_{\nu=1}^{n} \frac{r_{\nu}}{2} \cdot \frac{1+\varepsilon_{\nu} z}{1-\varepsilon_{\nu} z}\left(r_{\nu}>0,\left|\varepsilon_{\nu}\right|=1, k \leqq n\right)$, so that $a_{\nu}=r_{j} \varepsilon_{j}^{\nu}+\ldots+r_{k} \varepsilon_{k}^{\nu}$. We can prove the uniqueness of $k, r_{j} ; \varepsilon_{j}$ as follows. Suppose that $a_{\nu}$ be expressed in the form (22). We put $a_{0}=r_{1}+\ldots+t_{r} k$, then $\delta\left(a_{n}, a_{1}, \ldots, a_{n}\right)=0 . \quad$ Let $f(z)=\sum_{\nu=1}^{n} \frac{r_{\nu}}{2} \cdot \frac{1+\varepsilon_{\nu} z}{1-\varepsilon_{\nu} z}$, then $f(z)=\frac{a_{0}}{2}+a_{1} z+\ldots+a_{22} z^{2 n}\left(\bmod . z^{n+1}\right)$ and $\Re f(z) \geqq 0$ in $|z|<1$,

so that $H_{n}(x)=\sum_{0}^{n} a_{\mu-\nu} x_{\nu} \bar{x}_{\mu}$ is positive semi-definite, since $\delta\left(a, a_{1} \ldots, a_{n}\right)=0$. Hence $a_{0}$ is the greatest root of $\delta\left(x, a_{1}, \ldots, a_{n}\right)=0$, so that $a_{0}$ is unique. Since by Theorem 2 , such $f(z)$ as (23) is unique, $k, r_{j}, \varepsilon_{j}$ are unique.

7. Let $f(z)=\frac{a_{0}}{2}+\sum_{n=1}^{\infty} a_{n} z^{n}$ be regular in $|z|<1$, whose real part is not necessarily positive. We put

$$
m(\rho)=\operatorname{Min}_{|z|=\rho} \Re f(z), M(\rho)=\underset{|z|-\rho}{\operatorname{Max} .} \Re f(z)(0<\rho<1) .
$$

Then by $(14), g^{(P)} \leqq 2 m(\rho)$. Similarly $2 M(\rho) \leqq G^{(P)}$. On the other hand, from (8), $g_{i 2}^{(\rho)} \geqq 2 m(\rho)$, so that $g^{(\rho)} \geqq 2 m(\rho)$, hence $g^{(\rho)}=2 m(\rho)$. Similarly $G^{(\rho)}=2 M(\rho)$. Hence we have the theorem $:^{\text {क) }}$

Theorem 4. $g^{(\rho)}=2 m(\rho), G^{(P)}=2 M(\rho)$.

8) C, Carathéodory: l.c. (1). G. Szegö. 1.c. (1). I. Schur 1.c. (4). Frobenius 1.c. (4) M. Fujiwara: Über Entwicklungskoeffizienten der ralionalen Funktion. Proc. Phy.-Math. Soc. Japan. 3rd. series. 2 (1920).

9) C. Carathéodory und L. Fejér: Über den Zusamenhang der Extremen von harmonischen Funktionen mit ihrer Koeffizienten und über den Picard- Landauschen Satz: Rendiconti del circolo mat. Palermo. 32 (1911). 'O. Szaszl. c. (2). 